\title{
Attentional Disengagement Deficits Predict Brooding, but Not Reflection, Over a One-Year Period
}

\author{
Eric S. Allard* and llya Yaroslavsky* \\ Department of Psychology, Cleveland State University, Cleveland, $\mathrm{OH}$, United States
}

A growing literature suggests that rumination is linked to attentional disengagement deficits in depression. This is particularly the case with brooding, a maladaptive form of rumination. However, research on the potential constructive association between attentional disengagement and self-reflection, a putative adaptive form of rumination, is sparse. Thus, the goal of the present study was to examine whether visual attentional

OPEN ACCESS

Edited by: Alexandre Heeren, Catholic University of Louvain, Belgium

\section{Reviewed by: \\ Keisuke Takano, Ludwig Maximilian University \\ of Munich, Germany Matt R. Judah, Old Dominion University, United States \\ *Correspondence: Eric S. Allard e.s.allard@csuohio.edu llya Yaroslavsky \\ i.yaroslavsky@csuohio.edu}

Specialty section:

This article was submitted to Psychopathology,

a section of the journal

Frontiers in Psychology

Received: 29 July 2019 Accepted: 23 September 2019 Published: 14 October 2019

Citation:

Allard ES and Yaroslavsky I (2019) Attentional Disengagement Deficits Predict Brooding, but Not Reflection,

Over a One-Year Period.

Front. Psychol. 10:2282.

doi: 10.3389/fpsyg.2019.02282 disengagement deficits differentially predict dispositional brooding and self-reflection tendencies. Depressed participants $(n=17)$, those in remission from depression $(n=42)$, and their peers with no depression histories $(n=70)$ completed clinical interviews, the Ruminative Response Scale (RRS), and an eye-tracking task that measured attentional disengagement from pleasant (happy) and unpleasant (sad) facial images during a laboratory visit, and the RRS at 4 month intervals over a 1-year period. Results revealed that slow disengagement from sad faces, and rapid disengagement from happy faces, was specifically associated with brooding tendencies concurrently and across follow-up. Attentional disengagement was unrelated to self-reflection. The disengagement-brooding associations remained after controlling for depression status and anxiety disorder histories, suggesting that attentional control deficits may be a state-independent marker of brooding. Theoretical and clinical implications for these associations are discussed.

Keywords: attention, rumination, brooding, reflection, depression

\section{INTRODUCTION}

Failure to downregulate the intensity and duration of negative affective states, as appropriate to context, is a key vulnerability factor for depression and related psychopathology (Campbell-Sills and Barlow, 2007; Mennin et al., 2007; Joormann and Gotlib, 2010; Joormann and Quinn, 2014). Rumination, which reflects perseverative self-focused attention concerning the causes, meaning, and consequences of negative affect, has been shown to intensify and prolong negative affect and is a risk factor for depression (Nolen-Hoeksema, 2000; Spasojević and Alloy, 2001; NolenHoeksema et al., 2008). Indeed, findings from laboratory studies show that experimentally induced ruminative states maintain dysphoria following negative mood induction (Nolen-Hoeksema and Morrow, 1993; Blagden and Craske, 1996), and meta-analytic reviews show robust associations between rumination and depression severity (Aldao et al., 2010), as well as depression status 
(Visted et al., 2018). Further, ruminative tendencies endure after the remission of depressive episodes (Visted et al., 2018) and prognosticate a risk for episode recurrence (Kovacs et al., 2009, 2016; Michalak et al., 2011).

Rumination is not a unitary construct and is comprised of self-focused attentional modes that are differentially linked to depression and negative affect (Treynor et al., 2003; Verhaeghen et al., 2005; Burwell and Shirk, 2007; Rude et al., 2007). For instance, brooding - or self-critical moody pondering is considered maladaptive, as it is robustly associated with contemporaneous depression levels (Abela et al., 2002; Goodyer et al., 2003; Treynor et al., 2003; Watkins, 2004, 2009; Aldao et al., 2010; McLaughlin and Nolen-Hoeksema, 2011; Arnarson et al., 2016; Schäfer et al., 2017) and their worsening over time (Abela et al., 2002; Sarin et al., 2005; Roelofs et al., 2009; Calvete et al., 2015; Hudson et al., 2015; Gomez-Baya et al., 2017). Conversely, self-reflection - or the purposeful and non-judgmental appraisal of the positive or neutral content of distressing events - is believed to be an adaptive form of self-focused attention that facilitates problem solving (Treynor et al., 2003; Kross et al., 2005) and supports emotional wellbeing (Brans et al., 2013). Notably, the relationship between self-reflection and depression is less clear than that for brooding, with some studies finding no association between reflection and depression outcomes (Koval et al., 2012; Jose and Weir, 2013; Moore et al., 2013; Johnson et al., 2016; Tsypes and Gibb, 2016; Junkins and Haeffel, 2017; Artiran et al., 2019), others showing no distinction between its effects and those of brooding (Johnson and Whisman, 2013; Wilkinson et al., 2013; Padilla Paredes and Calvete Zumalde, 2015), and yet others observing the positive benefits of reflection on depressionrelevant processes (Joormann et al., 2006; Burwell and Shirk, 2007; Arditte and Joormann, 2011). Thus, while brooding and reflection are forms of self-focused attention, the two differ as to the target of salient focus and perhaps relate to divergent affective outcomes.

\section{Impaired Attentional Control, Brooding, Reflection, and Depression}

Some cognitive models of depression suggest that rumination (namely brooding) arises in part from attentional deficits or a reduced capacity to flexibly deploy and withdraw attention from internal and external sources generally concerning negative information when in a negative mood state (attentional disengagement; De Raedt and Koster, 2010; Whitmer and Gotlib, 2013). The "impaired disengagement hypothesis" in particular posits that attentional disengagement deficits have a synergistic relationship with brooding when confronted with a stressful event (Koster et al., 2011); such deficits result in a narrowed attentional focus that probalistically increase a brooding response that engenders further distress in lieu of a more effective strategy to manage distress (Koster et al., 2011). In a similar vein, the attentional scope model posits that dysphoric and low positive moods facilitate perseverative negative information processing concerning one's emotional state and problems (Whitmer and Gotlib, 2013).
Growing empirical evidence links impaired attentional disengagement with brooding. For instance, previous studies have observed behavioral evidence of impaired attentional disengagement when individuals are processing both nonemotional (Davis and Nolen-Hoeksema, 2000; De Lissnyder et al., 2011) and emotional stimuli (Joormann, 2006; Joormann and Gotlib, 2008; De Lissnyder et al., 2012a), and such deficits are related to dispositional brooding (Koster et al., 2011). Furthermore, difficulty switching attention away from negative emotional (and toward neutral) affective targets is linked with trait brooding (De Lissnyder et al., 2012b). Additional evidence from an affective probe discrimination task shows that brooding is associated with slow attention disengagement from negative distractor words (Southworth et al., 2017). Similarly, dispositional tendencies to brood are associated with sustained visual attention on sad faces, which indirectly suggests attentional inflexibility (Owens and Gibb, 2017). Finally, using an eye tracking task that delineated attentional engagement from disengagement in response to emotional and neutral valenced facial pairs, greater habitual brooding was predictive of slower disengagement from negative faces, particularly when experiencing high levels of daily stress (Sanchez-Lopez et al., 2019). Thus, perseverative processing of depressionrelevant stimuli (likely reflective of inflexible disengagement from negative affective elicitors) could be a key antecedent to brooding.

Though not fully articulated by either Koster's or Whitmer's model, a self-reflective response to negative self-relevant information may prove adaptive if the capacity to disengage attention from negative content when such information no longer poses a threat to well-being is intact. Empirical findings have associated self-reflection with enhanced attentional flexibility over self-relevant information (Daches et al., 2010), adaptive primary and secondary control strategies such as problem solving, cognitive restructuring, and acceptance (Burwell and Shirk, 2007), effective problem solving efforts (Haigh et al., 2018), and enhanced affective gains when problemsolving in daily life (Mori et al., 2015). As flexible attention is believed to support the effectiveness of adaptive coping and problem solving efforts (Ochsner and Gross, 2005; Teper and Inzlicht, 2013), there is indirect evidence for the role of attentional disengagement in the salubrious effects of selfreflection, which are well-aligned with the expected benefits of intact attentional disengagement that are proposed by the impaired disengagement hypothesis (Koster et al., 2011) and with a broadened attentional scope (Whitmer and Gotlib, 2013).

\section{Present Study}

Though considerable efforts have been made to test the role of attentional processes on depression risk and brooding, most investigations employ cross-sectional designs with non-clinical samples. Results from these studies, while providing insight into depression risk, are also limited by potential range restriction in brooding levels that are higher among clinical samples and by their inability to disambiguate whether their findings concern the trait or state component of rumination (see Bagby et al., 2004). Further, little is known concerning the potentially adaptive link between intact attentional disengagement capacities and 
self-reflection. The aims of the present study were therefore twofold: (1) to test whether attentional disengagement difficulties, indexed via an eye-tracking task measuring disengagement from depression-relevant stimuli (sad and happy faces; see Yaroslavsky et al., 2019), show differential contemporaneous associations with the brooding and self-reflection facets of depressive rumination in a mixed clinical sample of adults with various depression and anxiety disorder histories and (2) explore whether such relationships persist with the stable/invariant components of brooding and reflection across a 1-year period. Guided by conceptual work (Koster et al., 2011; Whitmer and Gotlib, 2013) and our prior findings that associate attentional disengagement deficits with depressive rumination (Yaroslavsky et al., 2019), we hypothesized that slow disengagement from sad faces, and rapid disengagement from happy faces, would positively predict contemporaneous and enduring brooding tendencies. Conversely, we hypothesized an inverse pattern of associations between the two attention disengagement indices and self-reflection. To contextualize our findings, we include the Ruminative Response Scale total score in our analyses from which the brooding and self-reflection indices arise, and test the clinical validity of the self-reflection index relative to contemporaneous depression symptoms and diagnostic status.

\section{METHODS}

\section{Participants}

One hundred twenty-nine participants ( $88 \%$ male, $\mathrm{M}_{\text {age }}=22.11$, $S D=8.16)$ with normal or corrected-to-normal vision were recruited through online advertisements, referrals from outpatient treatment facilities, and from an undergraduate psychology student subject pool. Though we sought to recruit a balanced sample of participants with $(n=70)$ and without depression histories $(n=70)$, which would enable us to detect small-to-medium effect sizes within general linear models (Cohen's $f^{2}=0.057$ ), our recruitment efforts were limited by budgetary constraints. Racial background of participants was 67\% Caucasian, 19\% African American, 6\% Latinx, 2\% Middle Eastern, 2\% multi-racial, and 4\% who endorsed the South Asian, South East Asian or "other" category. Fifty-nine participants $(46 \%)$ reported lifetime histories of a Depressive Disorder $(n=59$ Major Depressive Disorder, $n=3$ Dysthymic Disorder), of whom $n=17$ were in the midst of a depressive episode ( $n=15$ Major Depression, $n=2$ Dysthymic Disorder). Thirty (51\%) of those with a lifetime history of Depressive Disorders also reported lifetime anxiety disorder histories (34\% Social Anxiety Disorder, 6\% Specific Phobia, 4\% Obsessive Compulsive Disorder, 15\% Panic Disorder, 33\% Generalized Anxiety Disorder, 4\% Post Traumatic Stress Disorder). Of the participants $(n=70)$ with no Depressive Disorder histories, $n=19(27 \%)$ reported a lifetime history of anxiety disorders (16\% Social Anxiety Disorder, 3\% Specific Phobia, 2\% Obsessive Compulsive Disorder, 6\% Panic Disorder, 12\% Generalized Anxiety Disorder, 2\% Post Traumatic Stress Disorder), with the remaining $n=51$ participants denying lifetime histories of psychiatric disorders.

\section{Interview and Self-Report Measures Diagnostics Status}

Diagnostics status was ascertained via the Structured Clinical Interview of DSM-IV Disorders (SCID-I; First et al., 1994) by advanced graduate students and one of the authors (IY). The SCID-I is a well-validated measure of psychiatric disorders that evidenced good inter-rater reliability in this study (SCID-I, Fleiss' Кs $=0.73-0.90$ ). Diagnostic histories were determined during case consensus meetings following diagnostic consensus guidelines.

\section{Depression Symptoms}

Depression symptoms were measured via the self-rated Center for Epidemiological Depression Scale (CES-D; Radloff, 1977), a validated and reliable 20 -item measure of symptoms during the prior 1 week period ( $\alpha=0.92$ in this study).

\section{Rumination}

Rumination was measured via the Ruminative Response Scale (RRS), a 22-item survey of tendencies to brood and to reflect on one's negative mood state (Treynor et al., 2003). The Brooding and Reflection subscales of the RRS are each comprised of 5 items and respectively, measure tendencies toward moody pondering and non-judgmental self-evaluation. The RRS and its subscales are well validated and had good internal consistency properties in this study across observations (RRS: $\alpha s=0.93-0.96$; Brooding: $\alpha s=0.78-0.89$; Reflection: $\alpha s=0.70-0.83$ ).

\section{Eye Tracking Measures}

\section{Task and Stimuli}

The stimuli were face pairs that comprised emotional and neutral expressions. Faces were taken from the Karolinska Directed Emotional Faces (KDEF) database (Lundqvist et al., 1998) according to validation data from Sanchez et al. (2013). Based on a similar design from Sanchez et al. (2017), KDEF frontal view pictures that displayed discrete expressions of happiness, disgust, and sadness were used. A total of 24 happy, $24 \mathrm{sad}$, and 24 disgust face stimuli (12 men and 12 women for each emotion category, along with each actor's neutral expression stimulus) were selected for the eye tracking (ET) task.

The ET comprised 72 trials (24 happy-neutral, 24 sad-neutral, and 24 disgust-neutral pairs). Face stimuli were displayed on a $48 \mathrm{~cm}$ (width) $\times 27 \mathrm{~cm}$ (height) widescreen computer monitor. Each image was $12 \mathrm{~cm}$ (width) $\times 18 \mathrm{~cm}$ (height). Faces were centered on the screen, at a distance of $25 \mathrm{~cm}$ from the center of each image. Participants were approximately $60 \mathrm{~cm}$ from the center of the computer monitor. This resulted in a visual angle of approximately $11.8^{\circ}$ between each image's center and the center of the screen. The experimental design was similar to one used in Sanchez et al. (2013). Each trial started with a black screen for $500 \mathrm{~ms}$, followed by a central fixation cross for another $500 \mathrm{~ms}$. A single, random digit (i.e., 1-9) replaced the fixation cross and remained for 1,000 ms; participants were instructed to say this number aloud so as to ensure their attention was oriented to the center of the screen prior to the face pair presentation. Immediately after digit offset, the faces appeared on the screen for a 3,000-ms "free viewing" period (i.e., participants were instructed to "view the images naturally as if at home watching 
television"), after which a new trial began one-third of the time. Another third of the trials assessed attentional engagement with the emotional face in the pair: participants had to disengage gaze from the neutral face in order to engage gaze with the emotional face in the pair. The final third of the trials assessed attentional disengagement from the emotional face in the pair: participants had to disengage gaze from the emotional face in order to engage gaze with the neutral face in the pair. For the emotional engagement trials, after "free viewing," participants' fixation on the neutral face (for $100 \mathrm{~ms}$ ) triggered a rectangular or oval frame to appear around the opposite emotional face in the pair, and participants indicated the shape of the frame by pressing one of two keys on a keyboard corresponding to a "rectangle" or "oval." For the disengagement trials, the opposite occurred. In other words, a 100-ms fixation on the emotional face triggered a rectangle or oval frame to surround the neutral face, and disengagement was determined by the amount of time needed to shift gaze from the emotional face toward the neutral face. Participants completed two practice trials to ensure their understanding of task instructions. All three trial conditions were randomly presented, and both types of frames and valenced faces were equal in their presentation and whether or not they appeared in the left and right positions during the engagement and disengagement conditions. Emotional and neutral faces were equally presented on the left and right side of the screen across trials. A schematic example of a trial sequence can be found in Figure 1. The disengagement indices used in this study reflect the average delay (in ms) to first fixation on the target face (i.e., the face surrounded by a rectangle or oval), and were computed via Huber $\mathrm{M}$ estimation to down-weigh the influence of outliers (see Wilcox, 2017) comprising 1.5 and $1.7 \%$ of sad and happy face disengagement trials, respectively. Both disengagement indices displayed adequate internal consistency (Sad Disengagement $\alpha=0.68$, Happy Disengagement $\alpha=0.70$ ).

\section{Eye Tracking Apparatus}

Eye movements were recorded using the RED-m (Sensomotoric Instruments, SMI; Berlin, Germany) and Tobii X3-120 (Tobii Technology, Inc.) $120 \mathrm{~Hz}$ eye-tracking devices in the authors' labs, respectively. Both stimulus presentation and eye movement recording was conducted using SMI Experiment Center and E-prime v. 2 software. Visual fixations were defined as gaze resting within $0.5-1.0^{\circ}$ visual angle for at least $100 \mathrm{~ms}$ (Manor and Gordon, 2003) within pre-determined areas of interest (AOIs). AOIs comprised the entirety of the facial stimuli for both the free-viewing and engage-disengage tasks.

\section{Procedure}

Participants completed clinical evaluations, survey measures, an hour-long protocol that assessed physiological and psychological reactions to various stimuli/tasks, and the ET task during a fourhour laboratory session. They were then contacted 4-, 8-, and 12-months following their laboratory visit and completed a set of survey measures online that included the RRS. Time between follow-up assessments was approximately 4 months $(M s=3.66-$ 4.37, $S D s=0.88-1.01)$, with $n=102(79 \%)$ participants providing data on 2 occasions, $n=85(66 \%)$ on 3 occasions, and $n=72$ (56\%) completing all assessments.

\section{Statistical Analyses}

Descriptive and inferential analyses were conducted using SAS version 9.4 software (SAS Institute Inc., 2013). Pearson ChiSquare and Analyses of Variance (ANOVAs) that employed Tukey's HSD to correct for multiple comparisons were used to characterize the sample with respect to the three diagnostic groups. Bivariate correlations were calculated between clinical characteristics ascertained during the laboratory visit and contemporaneously measured Brooding and Reflection subscales of the RRS to test the clinical validity of the two subscales, as were regression models to test contemporaneous effects of attention indices on the Brooding and Reflection subscales, while covarying effects of demographic and clinical characteristics. Mixed effects models were employed to test associations between attention indices and time-invariant components of the RRS and its two subscales in order to accommodate the multi-level structure of the longitudinal data and were fit using Restricted Maximum Likelihood and empirical standard errors to accommodate moderate heteroscedasticity (Liang and Zeger, 1986). In these models, Level 1 dependent variables were the RRS and its subscales, which displayed high-tomoderate stability across follow-up assessments $\left(\mathrm{ICC}_{R R S}=0.73\right.$, $\left.\mathrm{ICC}_{\text {Brood }}=0.67, \mathrm{ICC}_{\text {Reflect }}=0.55, p s<0.001\right)$, and the two attention disengagement indices served as the Level 2 predictors of interest (Sad Dis. and Hap. Dis). Level 2 covariates included age and sex, as were depression status (C. Dep and P. Dep) and anxiety disorder histories $(A n x)$ in light of prior findings that associate attentional disengagement deficits with both disorder classes (Ellenbogen and Schwartzman, 2009; Reinholdt-Dunne et al., 2013). Continuous predictors and covariates were grand mean centered, while dichotomous predictors retained their original metric. The equations below reflect all fixed $(\gamma)$ and random (u) effects, with $\hat{Y}_{i j}$ reflecting the predicted value on the RRS, Brooding, or Reflection subscales on observation $i$ for participant $j$. Variance components along with first- and secondorder autoregressive structures were imposed on the random effects and residual variance-covariance matrices, respectively, to accommodate the single random effect (i.e., random intercept) and potential residual auto-correlations. However, the residual variance-covariance matrix was excluded from the final models to avoid model over-fitting, given that the inclusion of autoregressive parameters did not significantly improve model fit $(\Delta-2 \mathrm{LL}=0.4-2.20, p s=0.14-0.53$; Snijder and Bosker, 2012).

Level 1 equation

$$
\hat{Y}_{i j}=\beta_{0 j}
$$

Level 2 equations

$$
\begin{gathered}
\beta_{0 j}=\gamma_{00}+\gamma_{01} \text { Age }+\gamma_{02} \text { Sex }+\gamma_{03} \text { Anx }+ \\
\gamma_{04} \text { C.Dep }+\gamma_{05} \text { P.Dep }+\gamma_{06} \text { Sad Dis. }+\gamma_{07} \text { Hap.Dis. }+u_{0 j}
\end{gathered}
$$




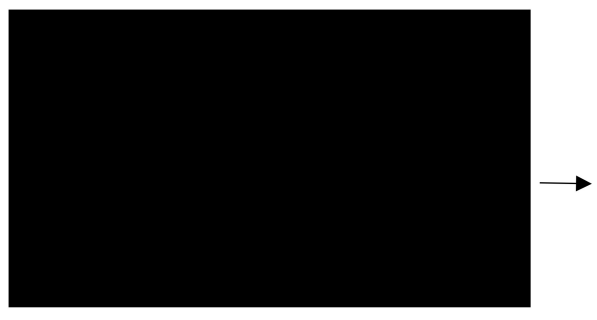

$500 \mathrm{~ms}$

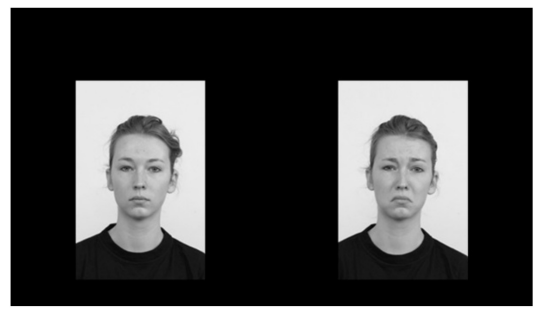

$3000 \mathrm{~ms}$

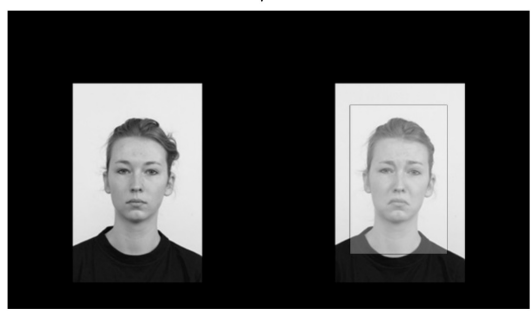

Until Fixation

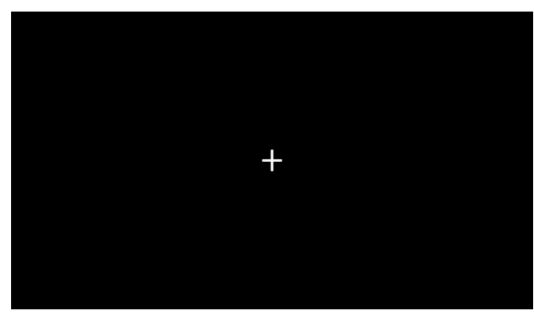

$500 \mathrm{~ms}$

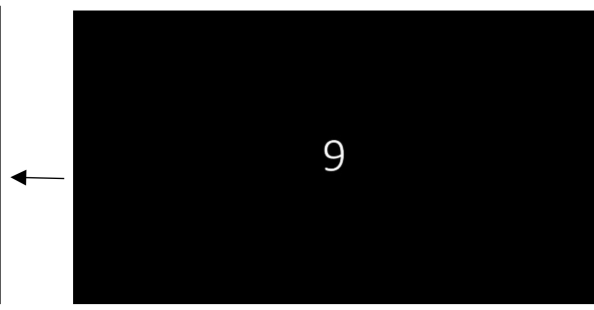

$1000 \mathrm{~ms}$

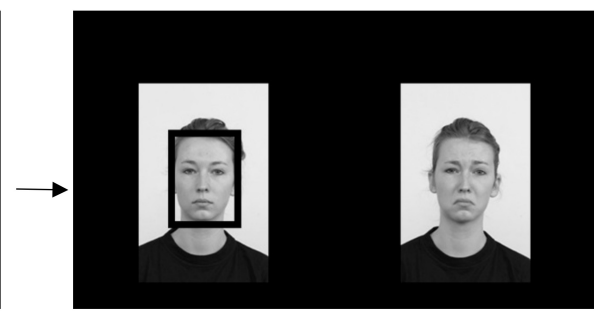

Until Response

FIGURE 1 | Schematic outline of the ET procedure. The arrows denote the temporal order of the stimulus presentation. Note that for one-third of the trials, the "Until Fixation" and "Until Response" stimuli were not presented. Figure reproduced with permission from Yaroslavsky et al. (2019).

\section{RESULTS}

\section{Preliminary Analyses}

Group differences in demographic, clinical, and attentional characteristics of depressed, remitted, and control participants are presented in Table 1. Depressed participants were significantly older than their remitted and control peers, but otherwise the three groups did not differ in their demographic characteristics. Groups evidenced a rank order in depression symptoms, with those in the midst of a depressive episode reporting the highest levels that were followed by remitted and control participants. Those in the remitted group evidenced more abundant histories of anxiety disorders than control participants, while depressed participants did not significantly differ from the other two groups. The three groups showed similar patterns of attentional disengagement from positive and negative valenced faces.

Consistent with the extant literature, scores on the Brooding subscales positively correlated with depression symptoms, $r(127)=0.57, p<0.001$, and anxiety disorder histories at a non-significant trend level, $r(127)=0.17, p=0.06$. Notably, the Reflection subscale scores also positively correlated with depression levels, $r(127)=0.38, p<0.001$, anxiety disorder histories, $r(127)=0.18, p=0.04$, and those of the Brooding subscale, $r(127)=0.64, p<0.001$. Therefore, contrary to expectation, the Reflection subscale did not evidence clinical validity, given its positive associations with indices of psychopathology nor distinction at the construct level from the Brooding subscale.

\section{Do Attentional Disengagement Deficits Predict Trait Rumination, Brooding, and Reflection?}

Results from regression and mixed effects models are presented in Tables 2, 3. As hypothesized, delayed disengagement from sad faces robustly predicted elevated tendencies to ruminate $\left(\beta_{R R S}=0.18, \gamma_{R R S}=0.036, p s=0.013-0.03\right)$ and to brood $\left(\beta_{\text {Brood }}=0.19, \gamma_{\text {Brood }}=0.01, p s=0.02-0.03\right)$ across the laboratory and follow-up assessments, as did rapid disengagement from happy faces $\left(\beta_{\text {Brood }}=-0.20, \gamma_{R R S}=-0.032\right.$, $p s=0.005-0.03 ; \beta_{\text {Brood }}=-0.23, \gamma_{\text {Brood }}=-0.033$, $p s=0.007-0.05)$. Importantly, these effects were independent of depression status and anxiety disorder histories, which significantly predicted both outcomes and suggest that the effects 
TABLE 1 | Demographic, clinical, and attentional characteristics among control, remitted, and depressed participants.

\begin{tabular}{|c|c|c|c|c|}
\hline Variable & Control $(n=70)$ & Remitted $(n=42)$ & Depressed $(n=17)$ & Test Statistic \\
\hline Age & $19.76(3.00)^{\mathrm{a}}$ & $24.17(9.74)^{\mathrm{a}}$ & $26.65(13.91)^{b}$ & $F(2,126)=7.58, p<0.001, \eta^{2}=0.11$ \\
\hline Sex (Female) & $67 \%$ & $76 \%$ & $52 \%$ & $\chi^{2}(2)=3.10, p=0.21, v=0.16$ \\
\hline $\operatorname{Race}^{\dagger}$ & & & & $\chi^{2}(4)=4.58, p=0.31, v=0.13$ \\
\hline African A & $14 \%$ & $22 \%$ & $29 \%$ & \\
\hline Caucasian & $72 \%$ & $64 \%$ & $71 \%$ & \\
\hline Other & $14 \%$ & $14 \%$ & $0 \%$ & \\
\hline Anxiety $\mathrm{Hx}$ & $27 \%^{a}$ & $55 \%{ }^{\mathrm{b}}$ & $41 \%$ a,b & $\chi^{2}(2)=8.59, p=0.01, V=0.26$ \\
\hline CES-D & $9.79(7.64)^{\mathrm{a}}$ & $17.07(9.20)^{\mathrm{b}}$ & $29.82(8.71)^{\mathrm{C}}$ & $F(2,126)=7.58, p<0.001, \eta^{2}=0.40$ \\
\hline Sad Disengagement & $246.99(65.16)$ & $271.47(94.74)$ & $251.55(76.24)$ & $F(2,126)=1.34, p=0.27, \eta^{2}=0.02$ \\
\hline Happy Disengagement & $246.79(54.93)$ & $259.32(71.86)$ & $241.15(60.04)$ & $F(2,126)=0.75, p=0.47, \eta^{2}=0.01$ \\
\hline
\end{tabular}

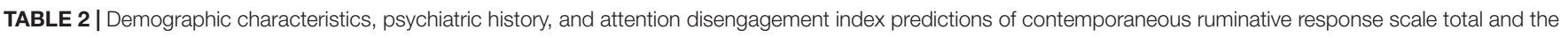
brooding and reflection subscales scores.

\begin{tabular}{|c|c|c|c|c|c|c|c|c|c|}
\hline \multirow[b]{2}{*}{ Variable } & \multicolumn{3}{|c|}{ RRS } & \multicolumn{3}{|c|}{ Brooding } & \multicolumn{3}{|c|}{ Reflection } \\
\hline & B & SE & $\beta$ & B & SE & $\beta$ & B & SE & $\beta$ \\
\hline Age & $-0.37^{*}$ & 0.16 & -0.21 & $-0.14^{* *}$ & 0.04 & -0.28 & -0.00 & 0.09 & -0.00 \\
\hline Sex & 1.21 & 2.38 & 0.04 & -0.01 & 0.67 & -0.00 & 0.62 & 0.57 & 0.08 \\
\hline Current dep. D/O & $14.42^{* * *}$ & 3.36 & 0.34 & $3.61^{* * *}$ & 0.93 & 0.31 & $2.09^{\dagger}$ & 1.10 & 0.21 \\
\hline Past dep. D/O & $5.54^{*}$ & 2.55 & 0.19 & $1.63^{*}$ & 0.69 & 0.20 & 0.54 & 0.68 & 0.08 \\
\hline Anx. D/O Hx & 4.13 & 2.48 & 0.14 & 0.82 & 0.70 & 0.10 & $1.15^{\dagger}$ & 0.62 & 0.16 \\
\hline Sad disengage & $0.033^{*}$ & 0.013 & 0.18 & $0.010^{*}$ & 0.004 & 0.19 & 0.004 & 0.004 & 0.09 \\
\hline Happy disengage & $-0.047^{* *}$ & 0.016 & -0.20 & $-0.015^{* *}$ & 0.005 & -0.23 & $-0.009^{\dagger}$ & 0.005 & -0.17 \\
\hline$R^{2}$ & & 0.25 & & & 0.26 & & & 0.12 & \\
\hline
\end{tabular}

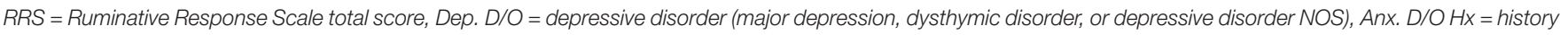

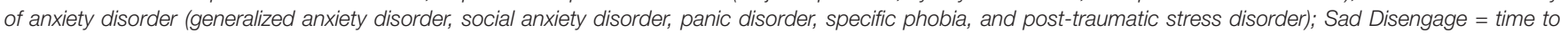

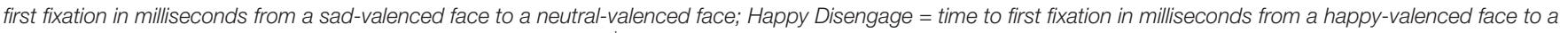
neutral-valenced face. ${ }^{* *} p \leq 0.001,{ }^{* *} p \leq 0.01,{ }^{*} p<0.05$, and ${ }^{\dagger} p<0.07$.

of attentional disengagement deficits are state-independent and enduring. Of note, and in contrast to expectation, neither disengagement from sad nor happy faces predicted trait reflection levels across the follow-up period, though akin to brooding, fast disengagement from happy faces predicted a contemporaneous tendency toward reflection at a trend level. Indeed, of the covariates, only depressive disorder histories evidenced a significant positive relationship with reflection levels, $\gamma=1.11$, $t(121)=2.04, p=0.04$.

\section{DISCUSSION}

Conceptual works and growing empirical evidence suggests that rumination, a well-known risk factor for depressive disorders (Nolen-Hoeksema et al., 2008), is associated with attentional disengagement deficits (Koster et al., 2011; Sanchez-Lopez et al., 2019; Yaroslavsky et al., 2019). However, not all ruminative thinking takes a depressogenic form (e.g., Brooding), as nonjudgmental self-reflection has been linked in some studies to emotional well-being and effective problem solving (Kross et al., 2005; Brans et al., 2013). As the preponderance of studies on the relationship between attentional disengagement deficits and rumination focus on the maladaptive forms of self-focused attention, the relationship between attentional flexibility and self-reflection is not clear. We sought to address this gap in the literature by testing whether slow visual attentional disengagement from unpleasant (sad faces) and rapid disengagement from pleasant (happy faces) stimuli differentially predict the adaptive and maladaptive forms of self-focused attention. To wit, we examined associations between attentional disengagement and the Brooding and Reflection facets of the Ruminative Response Scale, a common measure of depressive rumination, that were contemporaneously measured with attention disengagement and across 12 months in a sample of adults with various depressive and anxiety disorder histories.

As hypothesized, slow attentional disengagement from sad faces was significantly predictive of ruminative tendencies, particularly brooding, during the initial laboratory assessment 
TABLE 3 | Demographic characteristic, psychiatric history, and attention disengagement index predictions of invariant ruminative response scale total and the brooding and reflection subscales scores.

\begin{tabular}{lccc}
\hline Variable & RRS $\gamma$ (SE) & Brooding $\gamma$ (SE) & $\begin{array}{c}\text { Reflection } \\
\gamma \text { (SE) }\end{array}$ \\
\hline Age & $-0.32^{*}(0.14)$ & $-0.09^{* *}(0.03)$ & $-0.04(0.04)$ \\
Sex & $-0.54(2.04)$ & $0.10(0.55)$ & $-0.34(0.51)$ \\
Current dep. D/O & $12.88^{* * *}(3.26)$ & $3.56^{* * *}(0.99)$ & $1.11(0.95)$ \\
Past dep. D/O & $6.90^{* *}(2.34)$ & $1.55^{* *}(0.59)$ & $1.21^{*}(0.59)$ \\
Anx. D/O Hx & $4.85^{*}(2.24)$ & $1.25^{*}(0.58)$ & $0.78(0.55)$ \\
Sad disengage & $0.036^{*}(0.016)$ & $0.01^{*}(0.004)$ & $0.005(0.004)$ \\
Happy disengage & $-0.033^{*}(0.016)$ & $-0.011^{* *}(0.004)$ & $-0.002(0.005)$ \\
Random Effects & & & \\
Intercept & $119.45^{* * *}(18.74)$ & $7.56^{* * *}(1.26)$ & $6.84 * * *(1.19)$ \\
Residual & $60.57^{* * *}(5.43)$ & $5.07^{* * *}(0.45)$ & $5.98^{* * *}(0.53)$ \\
Pseudo $R^{2 a}$ & 0.26 & 0.27 & 0.05 \\
\hline
\end{tabular}

$R R S=$ Ruminative Response Scale total score, Dep. D/O = depressive disorder (major depression, dysthymic disorder, or depressive disorder NOS), Anx. D/O $H x=$ history of anxiety disorder (generalized anxiety disorder, social anxiety disorder, panic disorder, specific phobia, and post-traumatic stress disorder); Sad Disengage $=$ time to first fixation in milliseconds from a sad-valenced face to a neutral-valenced face; Happy Disengage = time to first fixation in milliseconds from a happy-valenced face to a neutral-valenced face. ${ }^{a}$ Pseudo $R^{2}$ approximates variance explained in the Random Intercept component by model predictors and covariates relative to the empty model. ${ }^{* * *} p \leq 0.001$, ${ }^{* *} p \leq 0.01$, and ${ }^{*} p<0.05$.

and over the course of the 1-year follow-up period, as was rapid disengagement from happy faces. These results are in line with prior work observing that slower disengagement from depression-relevant information is associated with greater levels of habitual brooding (Sanchez-Lopez et al., 2019). Additionally, the present results extend our prior findings as to the role of rapid disengagement from pleasant affective stimuli being linked with rumination (Yaroslavsky et al., 2019). This further evinces the role of attentional disengagement issues - in relation to pleasant and unpleasant affective elicitors - on the perseverative, negative self-relevant thoughts that are a hallmark of depressive symptomology (Koster et al., 2011). Importantly, the predictive associations between attentional disengagement and brooding were independent of depression status and anxiety disorder histories. This suggests that attentional disengagement problems may be a state-independent, and perhaps transdiagnostic, prognosticator of maladaptive selffocused attention.

Contrary to our hypotheses, no attentional disengagement indices were significantly predictive of self-reflection tendencies during the laboratory assessment or at follow-up. Thus, while flexible attentional disengagement has been shown to be adaptive in a variety of contexts (Ochsner and Gross, 2005; Teper and Inzlicht, 2013), the present study did not observe the salutary effects of flexible attentional disengagement on self-reflection. This was further evidenced by the lack of a significant correlation between the RRS Reflection subscale and depression levels in the present sample. Our results add to the mixed evidence for identifying the predictive relationships between self-reflection and depression-related outcomes (see Arditte and Joormann, 2011; Johnson and Whisman, 2013:
Padilla Paredes and Calvete Zumalde, 2015; Johnson et al., 2016; Artiran et al., 2019).

The potential trait-based role of positive and negative attentional disengagement in predicting brooding - rather than self-reflection - is a novel contribution that could inform theory and practice regarding cognitive models of depression. Recent research has observed associations between training paradigms for treating attentional disengagement problems in response to negative affective elicitors (e.g., Attentional Bias Modification), observing that attentional training can work to alleviate prospective depression symptoms (Beevers et al., 2015; Yang et al., 2015) and episodic recurrence (Browning et al., 2012). One lingering question with such training paradigms is the mechanism of action linking attentional modification and depressive outcomes. It is plausible that improvements to attentional disengagement processes are most influential by acting upon negative perseverative brooding styles. Future work is needed to better determine how improving attentional disengagement capacities impacts depressive outcomes through influences on rumination processes.

\section{LIMITATIONS AND FUTURE DIRECTIONS}

Though this study has several strengths in the form of a well-characterized clinical sample, the use of a novel eyetracking paradigm, and a longitudinal design, it is not without limitations. First, the sample was primarily comprised of Caucasian women, thereby limiting generalizability of our findings. Second, though attrition is a common problem in longitudinal studies, the reduction of our sample size limited the precision and power of our statistical analyses. Finally, though trait-like in its nature, ruminative thinking has been shown to fluctuate over time (Bagby et al., 2004). Due to attrition, we were unable to fit statistical models that could test the concomitant associations between attentional control indices and time-varying and invariant components of self-referential thinking. Future studies that recruit and retain a large and diverse clinical sample would do much to elucidate whether and in what way attentional disengagement differentially predicts the purported adaptive and maladaptive forms of rumination.

\section{CONCLUSION}

The present findings further highlight associations, based on cognitive models of depression, between attentional disengagement difficulties and rumination, with a key link emerging for brooding relative to self-reflection. The fact that these associations were independent of depression status and anxiety disorder histories suggest that such attentional disengagement deficits may be a trait-based marker of problematic affect that undermines attempts at well-being for individuals experiencing current episode depression, remitted depression, and even among those without a prior depression 
history. Thus, interventions that target the insidious link between attentional disengagement difficulties and brooding could be key for reducing depression symptoms, enhancing relapse prevention, and protecting against first episode occurrence.

\section{DATA AVAILABILITY STATEMENT}

Data for independent replication of our findings are available from the corresponding authors upon request.

\section{ETHICS STATEMENT}

The studies involving human participants were reviewed and approved by the Cleveland State University Institutional Review Board. The participants provided their written informed consent to participate in this study.

\section{REFERENCES}

Abela, J. R. Z., Brozina, K., and Haigh, E. P. (2002). An examination of the response styles theory of depression in third- and seventh-grade children: a short-term longitudinal study. J. Abnorm. Child Psychol. 30, 515-527. doi: 10.1023/A: 1019873015594

Aldao, A., Nolen-Hoeksema, S., and Schweizer, S. (2010). Emotion-regulation strategies across psychopathology: a meta-analytic review. Clin. Psychol. Rev. 30, 217-237. doi: 10.1016/j.cpr.2009.11.004

Arditte, K. A., and Joormann, J. (2011). Emotion regulation in depression: reflection predicts recovery from a major depressive episode. Cog. Ther. Res. 35, 536-543. doi: 10.1007/S110608-011-9389-4

Arnarson, E. Ö, Matos, A. P., Salvador, C., Ribeiro, C., de Sousa, B., and Edward Craighead, W. (2016). Longitudinal study of life events, well-being, emotional regulation and depressive symptomatology. J. Psychopathol. Behav. Assess. 38, 159-171. doi: 10.1007/s10862-015-9524-8

Artiran, M., Simsek, O. F., and Turner, M. (2019). Mediational role of rumination and reflection on irrational beliefs and distress. Behav. Cogn. Psychother. doi: 10.1017/S1352465819000031 [Epub ahead of print].

Bagby, R. M., Rector, N. A., Bacchiochi, J. R., and McBride, C. (2004). The stability of the response styles questionnaire rumination scale in a sample of patients with major depression. Cogn. Ther. Res. 28, 527-538. doi: 10.1023/B:COTR. 0000045562.17228 .29

Beevers, C. G., Clasen, P. C., Enock, P. M., and Schnyer, D. M. (2015). Attention bias modification for major depressive disorder: effects on attention bias, resting state connectivity, and symptom change. J. Abnorm. Psychol. 124, 463-475. doi: 10.1037/abn0000049

Blagden, J. C., and Craske, M. G. (1996). Effects of active and passive rumination and distraction: a pilot replication with anxious mood. J. Anxiety Disord. 10, 243-252. doi: 10.1016/0887-6185(96)00009-6

Brans, K., Koval, P., Verduyn, P., Lim, Y. L., and Kuppens, P. (2013). The regulation of negative and positive affect in daily life. Emotion 13, 926-939. doi: 10.1037/ a0032400

Browning, M., Holmes, E. A., Charles, M., Cowen, P. J., and Harmer, C. J. (2012). Using attentional bias modification as a cognitive vaccine against depression. Biol. Psychiatry 72, 572-579. doi: 10.1016/j.biopsych.2012.04.014

Burwell, R. A., and Shirk, S. R. (2007). Subtypes of rumination in adolescence: associations between brooding, reflection, depressive symptoms, and coping. J. Clin. Child Psychol. 36, 56-65. doi: 10.1080/15374410709336568

Calvete, E., Orue, I., and Hankin, B. L. (2015). Cross-lagged associations among ruminative response style, stressors, and depressive symptoms in adolescents. J. Soc. Clin. Psychol. 34, 203-220. doi: 10.1521/jscp.2015.34.3.203

Campbell-Sills, L., and Barlow, D. H. (2007). "Incorporating emotion regulation into conceptualizations and treatments of anxiety and mood disorders," in

\section{AUTHOR CONTRIBUTIONS}

EA and IY contributed equally to the design and execution of the study protocol, as well as all aspects of manuscript preparation.

\section{FUNDING}

This work was supported in part by Cleveland State University's Undergraduate Summer Research Award (PI: IY).

\section{ACKNOWLEDGMENTS}

The authors thank the Aging, Cognition, and Emotion Laboratory and the Mood and Emotion Regulation Laboratory for help with data collection.

Handbook of Emotion Regulation, ed. J. J. Gross (New York, NY: The Guilford Press), 542-559.

Daches, S., Mor, N., Winquist, J., and Gilboa-Schechtman, E. (2010). Brooding and attentional control in processing self-encoded information: evidence from a modified garner task. Cogn. Emot. 24, 876-885. doi: 10.1080/ 02699930902954825

Davis, R. N., and Nolen-Hoeksema, S. (2000). Cognitive inflexibility among ruminators and nonruminators. Cog. Ther. Res. 24, 699-711. doi: 10.1016/j. actpsy.2013.01.008

De Lissnyder, E., Derakshan, N., De Raedt, R., and Koster, E. H. (2011). Depressive symptoms and cognitive control in a mixed antisaccade task: specific effects of depressive rumination. Cogn. Emot. 25, 886-897. doi: 10.1080/02699931.2010. 514711

De Lissnyder, E., Koster, E. H. W., and De Raedt, R. (2012a). Emotional interference in working memory is related to rumination. Cog. Ther. Res. 36, 348-357. doi: 10.1007/s10608-011-9352-4

De Lissnyder, E., Koster, E. H. W., Goubert, L., Onraedt, T., Vanderhasselt, M. A., and De Raedt, R. (2012b). Cognitive control moderates the association between stress and rumination. J. Behav. Ther. Exp. Psychiatry 43, 519-525. doi: 10.1016/ j.jbtep.2011.07.004

De Raedt, R., and Koster, E. H. W. (2010). Understanding vulnerability for depression from a cognitive neuroscience perspective: a reappraisal of attentional factors and a new conceptual framework. Cogn. Affect. Behav. Neurosci. 10, 50-70. doi: 10.3758/CABN. 10.1 .50

Ellenbogen, M. A., and Schwartzman, A. E. (2009). Selective attention and avoidance on a pictorial cueing task during stress in clinically anxious and depressed participants. Beh. Res. Ther. 47, 128-138. doi: 10.1016/j.brat.2008.1 0.021

First, M. B., Spitzer, R. L., Gibbon, M., and Williams, J. B. W. (1994). Structured clinical Interview for DSM-IV-TR Axis I Disorders, Research Version, Nonpatient Edition. New York, NY: Biometric Research Department.

Gomez-Baya, D., Mendoza, R., Paino, S., Sanchez, A., and Romero, N. (2017). Latent growth curve analysis of gender differences in response styles and depressive symptoms during mid-adolescence. Cogn. Ther. Res. 41, 289-303. doi: 10.1007/s10608-016-9822-9

Goodyer, I. M., Herbert, J., and Tamplin, A. (2003). Psychoendocrine antecedents of persistent first-episode major depression in adolescents: a communitybased longitudinal enquiry. Psychol. Med. 33, 601-610. doi: 10.1017/ S0033291702007286

Haigh, E. A. P., Quinones, V., and Moore, M. T. (2018). Structural validity of the social problem-solving inventory-revised short form: application to brooding and reflection. J. Cogn. Psychother. 32, 285-302. doi: 10.1891/0889-8391.32. 4.285 
Hudson, M. R., Harding, K. A., and Mezulis, A. (2015). Dampening and brooding jointly link temperament with depressive symptoms: a prospective study. Pers. Individ. Dif. 83, 249-254. doi: 10.1016/j.paid.2015.04.025

Johnson, D. P., Rhee, S. H., Friedman, N. P., Corley, R. P., Munn-Chernoff, M. A., Hewitt, J. K., et al. (2016). A twin study examining rumination as a transdiagnostic correlate of psychopathology. Clin. Psychol. Sci. 4, 971-987. doi: $10.1177 / 2167702616638825$

Johnson, D. P., and Whisman, M. A. (2013). Gender differences in rumination: a meta-analysis. Pers. Individ. Dif. 55, 367-374. doi: 10.1016/j.paid.2013.03.019

Joormann, J. (2006). Differential effects of rumination and dysphoria on the inhibition of irrelevant emotional material: evidence from a negative priming task. Cog. Ther. Res. 30, 149-160. doi: 10.1007/s10608-006-9035-8

Joormann, J., Dkane, M., and Gotlib, I. H. (2006). Adaptive and maladaptive components of rumination? diagnostic specificity and relation to depressive biases. Behav. Ther. 37, 269-280. doi: 10.1016/J.BETH.2006.01.002

Joormann, J., and Gotlib, I. H. (2008). Updating the contents of working memory in depression: interference from irrelevant negative material. J. Abornm. Psychol. 117, 182-192. doi: 10.1037/0021-843X.117.1.182

Joormann, J., and Gotlib, I. H. (2010). Emotion regulation in depression: relation to cognitive inhibition. Cogn. Emot. 24, 281-298. doi: 10.1080/ 02699930903407948

Joormann, J., and Quinn, M. E. (2014). Cognitive processes and emotion regulation in depression. Depress. Anxiety. 31, 308-315. doi: 10.1002/da.22264

Jose, P. E., and Weir, K. F. (2013). How is anxiety involved in the longitudinal relationship between brooding rumination and depressive symptoms in adolescents? J. Youth Adolesc. 42, 1210-1222. doi: 10.1007/s10964-012-9891-3

Junkins, M. B., and Haeffel, G. J. (2017). Rumination: reflection can amplify the depressogenic effects of brooding. Int. J. Cogn. Ther. 10, 34-46. doi: 10.1521/ ijct_2016_09_19

Koster, E. H., De Lissnyder, E., Derakshan, N., and De Raedt, R. (2011). Understanding depressive rumination from a cognitive science perspective: the impaired disengagement hypothesis. Clin. Psychol. Rev. 31, 138-145. doi: 10.1016/j.cpr.2010.08.005

Kovacs, M., Obrosky, S., and George, C. (2016). The course of major depressive disorder from childhood to young adulthood: recovery and recurrence in a longitudinal observational study. J. Affect. Disord. 203, 374-381. doi: 10.1016/j. jad.2016.05.042

Kovacs, M., Rottenberg, J., and George, C. (2009). Maladaptive mood repair responses distinguish young adults with early-onset depressive disorders and predict future depression outcomes. Psychol. Med. 39, 1841-1854. doi: 10.1017/ S0033291709005789

Koval, P., Kuppens, P., Allen, N. B., and Sheeber, L. (2012). Getting stuck in depression: the roles of rumination and emotional inertia. Cogn. Emot. 26, 1412-1427. doi: 10.1080/02699931.2012.667392

Kross, E., Ayduk, O., and Mischel, W. (2005). When asking "why" does not hurt: distinguishing rumination from reflective processing of negative emotions. Psychol. Sci. 16, 709-715. doi: 10.1111/j.1467-9280.2005.01600.x

Liang, K. Y., and Zeger, S. L. (1986). Longitudinal data analysis using generalized linear models. Biometrika 73, 13-22. doi: 10.1093/biomet/73.1.13

Lundqvist, D., Flykt, A., and Öhman, A. (1998). The Karolinska Directed Emotional Faces-KDEF (CD-ROM). Stockholm: Karolinska Institute.

Manor, B. R., and Gordon, E. (2003). Defining the temporal threshold for ocular fixation in free-viewing visuocognitive tasks. J. Neurosci. Methods 128, 85-93. doi: 10.1016/S0165-0270(03)00151-1

McLaughlin, K. A., and Nolen-Hoeksema, S. (2011). Rumination as a transdiagnostic factor in depression and anxiety. Behav. Res. Ther. 49, 186-193. doi: 10.1016/j.brat.2010.12.006

Mennin, D. S., Holaway, R. M., Fresco, D. M., Moore, M. T., and Heimberg, R. G. (2007). Delineating components of emotion and its dysregulation in anxiety and mood psychopathology. Behav. Ther. 38, 284-302. doi: 10.1016/j.beth.2006. 09.001

Michalak, J., Hölz, A., and Teismann, T. (2011). Rumination as a predictor of relapse in mindfulness-based cognitive therapy for depression. Psychol. Psychother. 84, 230-236. doi: 10.1348/147608310X520166

Moore, M. N., Salk, R. H., Van Hulle, C. A., Abramson, L. Y., Hyde, J. S., LemeryChalfant, K., et al. (2013). Genetic and environmental influences on rumination, distraction, and depressed mood in adolescence. Clin. Psychol. Sci. 1, 316-322. doi: $10.1177 / 2167702612472884$
Mori, M., Takano, K., and Tanno, Y. (2015). Role of self-focus in the relationship between depressed mood and problem solving. Motiv. Emot. 39, 827-838. doi: 10.1007/s11031-015-9486-x

Nolen-Hoeksema, S. (2000). The role of rumination in depressive disorders and mixed anxiety/depressive symptoms. J. Abnorm. Psychol. 109, 504-511. doi: 10.1037//0021-843x.109.3.504

Nolen-Hoeksema, S., and Morrow, J. (1993). Effects of rumination and distraction on naturally occurring depressed mood. Cogn. Emot. 7, 561-570. doi: 10.1080/ 02699939308409206

Nolen-Hoeksema, S., Wisco, B. E., and Lyubomirsky, S. (2008). Rethinking rumination. Perspect. Psychol. Sci. 3, 400-424. doi: 10.1111/j.1745-6924.2008. 00088.x

Ochsner, K. N., and Gross, J. J. (2005). The cognitive control of emotion. Trends Cogn. Sci. 9, 242-249. doi: 10.1016/j.tics.2005.03.010

Owens, M., and Gibb, B. E. (2017). Brooding rumination and attentional biases in currently non-depressed individuals: an eye-tracking study. Cogn. Emot. 31, 1062-1069. doi: 10.1080/02699931.2016.1187116

Padilla Paredes, P., and Calvete Zumalde, E. (2015). A test of the vulnerabilitystress model with brooding and reflection to explain depressive symptoms in adolescence. J. Youth Adolesc. 44, 860-869. doi: 10.1007/s10964-0140148-1

Radloff, L. S. (1977). The CES-D scale: a self-report depression scale for research in the general population. Appl. Psychol. Meas. 1, 385-401. doi: 10.1177/ 014662167700100306

Reinholdt-Dunne, M. L., Mogg, K., and Bradley, B. P. (2013). Attention control: relationships between self-report and behavioural measures, and symptoms of anxiety and depression. Cogn. Emot. 27, 430-440. doi: 10.1080/02699931.2012. 715081

Roelofs, J., Rood, L., Meesters, C. M. G., te Dorsthorst, V., Bögels, S. M., Alloy, L. B., et al. (2009). The influence of rumination and distraction on depressed and anxious mood: a prospective examination of the response styles theory in children and adolescents. Euro. Child Adolesc. Psychiatry 18, 635-642. doi: 10.1007/s00787-009-0026-7

Rude, S. S., Maestes, K. L., and Neff, K. (2007). Paying attention to distress: what's wrong with rumination? Cogn. Emot. 21, 843-864. doi: 10.1080/ 02699930601056732

Sanchez, A., Romero, N., and De Raedt, R. (2017). Depression-related difficulties disengaging from negative faces are associated with sustained attention to negative feedback during social evaluation and predict stress recovery. PLoS One 12:e0175040. doi: 10.1371/journal.pone.0175040

Sanchez, A., Vázquez, C., Marker, C., LeMoult, J., and Joormann, J. (2013). Attentional disengagement predicts stress recovery in depression: an eyetracking study. J. Abnorm. Psychol. 122, 303-313. doi: 10.1037/a0031529

Sanchez-Lopez, A., Koster, E. H. W., Van Put, J., and De Raedt, R. (2019). Attentional disengagement from emotional information predicts future depression via changes in ruminative brooding: a five-month longitudinal eye-tracking study. Behav. Res. Ther. 118, 30-42. doi: 10.1016/j.brat.2019. 03.013

Sarin, S., Abela, J. R. Z., and Auerbach, R. P. (2005). The response styles theory of depression: a test of specificity and causal mediation. Cogn. Emot. 19, 751-761. doi: 10.1080/02699930441000463

Schäfer, J. Ö, Naumann, E., Holmes, E. A., Tuschen-Caffier, B., and Samson, A. C. (2017). Emotion regulation strategies in depressive and anxiety symptoms in youth: a meta-analytic review. J. Youth Adolesc. 46, 261-276. doi: 10.1007/ s10964-016-0585-0

Snijder, T. A. B., and Bosker, R. J. (2012). Multilevel Analysis: An Introduction to Basic and Advanced Multilevel Modeling, 2nd Edn. Thousand Oaks, CA: Sage Publications Ltd.

Southworth, F., Grafton, B., MacLeod, C., and Watkins, E. (2017). Heightened ruminative disposition is associated with impaired attentional disengagement from negative relative to positive information: support for the "impaired disengagement" hypothesis. Cogn. Emot. 31, 422-434. doi: 10.1080/02699931. 2015.1124843

Spasojević, J., and Alloy, L. B. (2001). Rumination as a common mechanism relating depressive risk factors to depression. Emotion 1, 25-37. doi: 10.1037/ /1528-3542.1.1.25

Teper, R., and Inzlicht, M. (2013). Meditation, mindfulness and executive control: the importance of emotional acceptance and brain-based performance 
monitoring. Soc. Cogn. Affect. Neurosci. 8, 85-92. doi: 10.1093/scan/ nss045

Treynor, W., Gonzalez, R., and Nolen-Hoeksema, S. (2003). Rumination reconsidered: a psychometric analysis. Cogn. Ther. Res. 27, 247-259.

Tsypes, A., and Gibb, B. E. (2016). Cognitive vulnerabilities and development of suicidal thinking in children of depressed mothers: a longitudinal investigation. Psychiatry Res. 239, 99-104. doi: 10.1016/j.psychres.2016.03.008

Verhaeghen, P., Joormann, J., and Khan, R. (2005). Why we sing the blues: the relation between self-reflective rumination, mood, and creativity. Emotion 5, 226-232. doi: 10.1037/1528-3542.5.2.226

Visted, E., Vøllestad, J., Nielsen, M. B., and Schanche, E. (2018). Emotion regulation in current and remitted depression: a systematic review and meta-analysis. Front. Psychol. 9:756. doi: 10.3389/fpsyg.2018.00756

Watkins, E. (2004). Adaptive and maladaptive ruminative self-focus during emotional processing. Behav. Res. Ther. 42, 1037-1052. doi: 10.1016/j.brat. 2004.01.009

Watkins, E. (2009). Depressive rumination and co-morbidity: evidence for brooding as a transdiagnostic process. J. Ration. Emot. Cogn. Behav. Ther. 27, 160-175. doi: 10.1007/s10942-009-0098-9

Whitmer, A. J., and Gotlib, I. H. (2013). An attentional scope model of rumination. Psychol. Bull. 139, 1036-1061. doi: 10.1037/a0030923

Wilcox, R. R. (2017). Introduction to Robust Estimation and Hypothesis Testing, 4th Edn. Burlington, MA: Elsevier.
Wilkinson, P. O., Croudace, T. J., and Goodyer, I. M. (2013). Rumination, anxiety, depressive symptoms and subsequent depression in adolescents at risk for psychopathology: a longitudinal cohort study. BMC Psychiatry 13:250. doi: 10.1186/1471-244X-13-250

Yang, W., Ding, Z., Peng, F., Zhang, J. X., and Dai, T. (2015). Attention bias modification training in individuals with depressive symptoms: a randomized controlled trial. J. Behav. Ther. Exp. Psychiatry 49, 101-111. doi: 10.1016/j.jbtep. 2014.08.005

Yaroslavsky, I., Allard, E. S., and Sanchez-Lopez, A. (2019). Can't look away: attention control deficits predict rumination, depression symptoms and depressive affect in daily life. J. Affect. Disord. 245, 1061-1069. doi: 10.1016/ j.jad.2018.11.036

Conflict of Interest: The authors declare that the research was conducted in the absence of any commercial or financial relationships that could be construed as a potential conflict of interest.

Copyright (c) 2019 Allard and Yaroslavsky. This is an open-access article distributed under the terms of the Creative Commons Attribution License (CC BY). The use, distribution or reproduction in other forums is permitted, provided the original author(s) and the copyright owner(s) are credited and that the original publication in this journal is cited, in accordance with accepted academic practice. No use, distribution or reproduction is permitted which does not comply with these terms. 